\title{
Is there an increased risk of twinning after discontinuation of the oral contraceptive pill?
}

\author{
M F G MURPHY, ${ }^{1}$ M J CAMPBELL ${ }^{2}$ AND M BONE ${ }^{3}$ \\ From ' the Department of Community Medicine and General Practice, Radcliffe Infirmary, Oxford; ${ }^{2}$ the \\ Department of Medical Statistics and Computing, General Hospital, Southampton; and ${ }^{3}$ the Social Survey \\ Division, OPCS, Kingsway, London.
}

ABSTRACT We investigated whether conception soon after cessation of use of oral contraceptives affects the chance of bearing twins. Unpublished data from a large sample survey of contraceptive practice in Scotland were used to derive oral contraceptive discontinuation rates by month over the decade 1972-82 and these were related to estimated monozygotic and dizygotic twin birth rates and proportions by month in Scotland from 1974-82, using regression techniques. The results provide some support for the hypothesis that there is an increased risk of conceiving monozygotic twins within a year of ceasing to use oral contraceptives.

The incidence of twinning in England and Wales rose from 1938 until the early 1950s, fell subsequently until the late 1970s and has since increased. Similar patterns have been observed worldwide. ${ }^{2}$ In most of these analyses, trends were estimated separately for monozygotic and dizygotic twins using Weinberg's method. ${ }^{3}$ The general finding is of secular change confined largely to dizygotic twinning, with monozygotic twinning stable or latterly increasing. ${ }^{4}$ While demographic change may account for part of these trends, the phenomenon is poorly understood, ${ }^{56}$ though circumstantial evidence incriminates the use of oral contraceptives in some countries. The availability of data on oral contraceptive discontinuation rates from a survey of family planning has allowed us to consider the relationship with subsequent twinning in Scotland.

\section{Methods}

In 1982 during a large sample survey of family planning among women in Scotland aged 16-54, 3410 women were successfully interviewed about their contraceptive practice over the previous ten years. ${ }^{7}$ It was known that a decline in prevalence of oral contraceptive use amongst women aged 15-44 occurred following publicity in 1976 about cardiovascular disease risks in oral contraceptive users. To discover whether this decline in prevalence was due to a rise in discontinuation rates. a reduction in acceptance rates or both, monthly acceptance and discontinuation rates were calculated by a life table method for the period $1972-82 .{ }^{8}$ The rates were also seasonally adjusted using the American Bureau of
Census XII computer program. We related the discontinuation rates of oral contraceptives $(\mathrm{OC})$ to subsequent monthly mono- and dizygotic twinning rates and proportions in Scotland. Because the zygosity of multiple births is not recorded at live or stillbirth registration, we first estimated numbers of identical and non-identical twins using Weinberg's method. ${ }^{3}$ This assumes non-identical twins to consist of equal numbers of like and unlike sexed pairs and the identical twins to comprise the remaining like sexed twins. If the total number of twin pairs born in month $t$ is $a_{t}$ and the number of unlike sexed twin pairs is $b_{t}$, on Weinberg's assumption the number of dizygotic twin pairs is $2 b_{t}$ and of monozygotic twin pairs $c_{t}=a_{t}-2 b_{t}$. On no occasion with our data did $2 b_{t}$ exceed $a_{t}$, so estimation of the respective numbers was possible for every month in the study. Seasonality of twinning was investigated using a modification of Edward's method. ${ }^{9}$

We regressed the estimated monthly monozygotic, dizygotic and total twinning counts for 1974-82 on the preceding monthly $O C$ discontinuation rates for 1972-1982 using the computer package GLIM ${ }^{10}$ and a number of models: (1) estimated monozygotic pair counts $c_{t}$ as a binomial random variable out of a sample size $a_{t} ;(2)$ the monthly monozygotic, dizygotic and total twin pair counts as Poisson random variables with mean $m_{t} \lambda_{t}$, where $m_{t}$ is the total number of confinements in month $t$, and $\lambda_{t}$ depends on the preceding OC discontinuation rate; and (3) a refinement of (2) (see appendix) to analyse the relationship between the probability of twins following the fertilization of a single egg and the preceding $\mathrm{OC}$ discontinuation rates. 
Extra-binomial variation was accounted for in (1) with a method described by Williams, ${ }^{11}$ and extraPoisson variation with the modification described by Breslow. ${ }^{12}$ For valid tests of significance, the residuals from the fitting process should plausibly be independent and this was investigated using the BoxPierce portmanteau statistic. ${ }^{13}$

\section{Results}

Figure 1 shows time trends in like sexed, unlike sexed and total twinning rates in Scotland 1952-84. There is a secular decline, with a recent upturn apparently contributed by like sexed twins in particular.

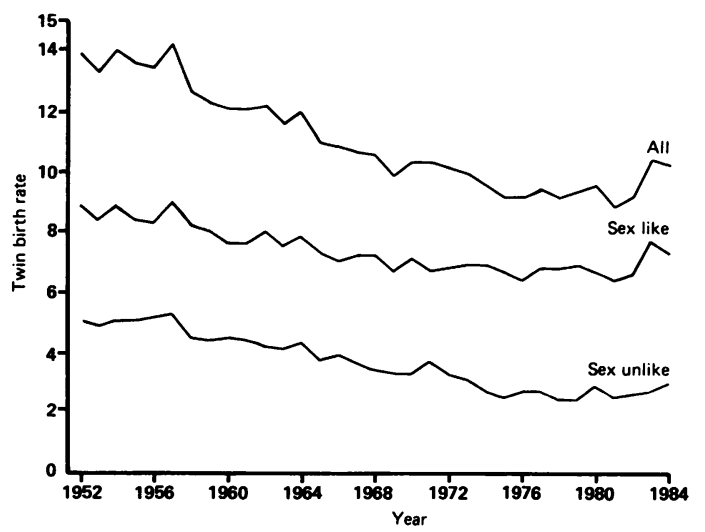

Fig 1. Total, like sexed and unlike sexed twinning rates per 1000 total confinements, 1952-84, Scotland.

Figure 2 shows $\mathrm{OC}$ discontinuation rates by month 1972-82. The rising discontinuation rates can clearly be seen from 1976-80, particularly in the seasonally adjusted trend.

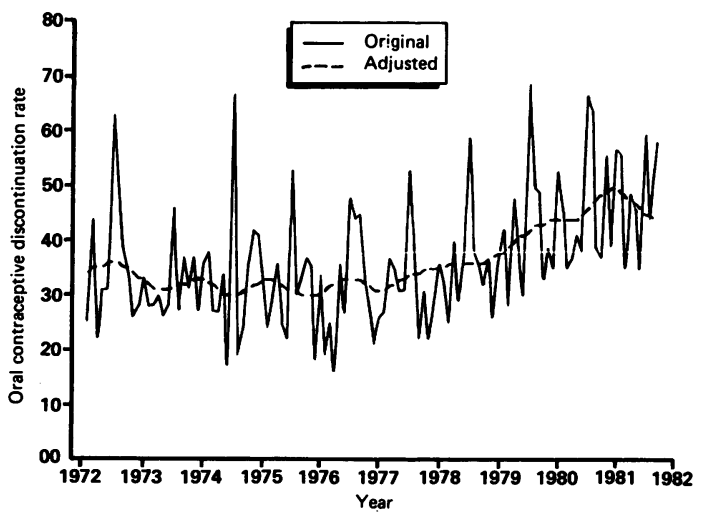

Fig. 2. Oral contraceptive pill discontinuation rates by month, 1972-82, Scotland.
Figure 3 shows estimated monthly monozygotic twinning rates 1974-82. There is a markedly low outlying rate in July 1977, but no apparent secular trend, and no evidence of serial correlation. $\left(\chi_{15}^{2}=19 \cdot 8, \mathrm{p}>0 \cdot 4\right)$.

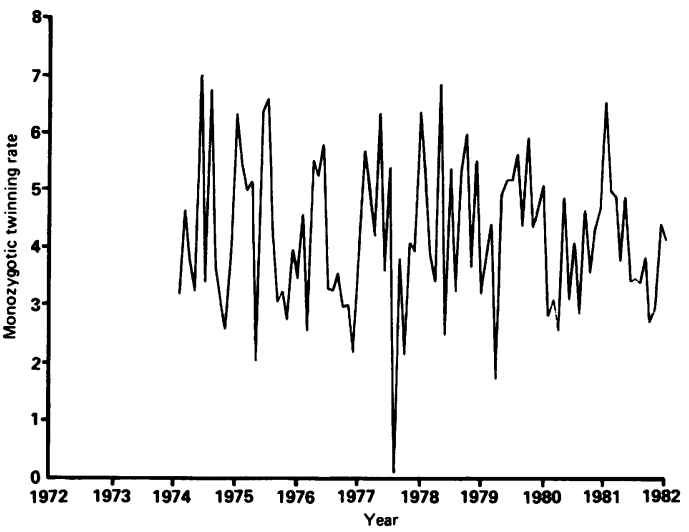

Fig 3. Estimated monozygotic twinning rate per 1000 total confinements, 1974-82, Scotland.

Testing the monozygotic, dizygotic and total twinning rates revealed no evidence of seasonality. We expected OC discontinuation rates to correlate with subsequent total confinements, and found two 0 significant positive correlations at lags of 13 months $(\mathrm{z}=2.55, \mathrm{p}=0.01)$ and 16 months $(\mathrm{z}=2.99, \mathrm{p}=0.003)$. When we looked at the relation between seasonally adjusted OC discontinuation rates and subsequent relative proportions of estimated monozygotic and dizygotic twins there was no significant relationship with the proportion dizygotic. As the table shows, however, an increase in the rate of $\mathrm{OC}$ discontinuation appears to be associated with the proportion of monozygotic twins 16-18 months later, though just failing to reach conventional statistical significance. There was no evidence of serial correlation of the residuals $\left(\chi_{15}^{2}=14.8, p>0 \cdot 5\right)$ so the test of significance for the coefficients is valid. For twinning rates per 1000 confinements, there was again no relationship between OC discontinuation rates and dizygotic or total twinning, but as the table shows there was a statistically significant relationship after a lag of 18 months for monozygotic twins. The more accurate non-linear regression of the $\mathrm{OC}$ discontinuation rates and the probability of twins following fertilisation of a single egg also showed a single significant positive correlation after a lag of 18 months. After excluding the outlying monozygotic rate of July 1977 the relationship remains significant $\left(\chi_{1}^{2}=4 \cdot 68, p=0.03\right)$. 
Table 1 Associations between oral contraceptive discontinuation rates and monozygotic twinning. Regression coefficients from logistic or log-linear modelling. $\left(\times 10^{3}\right)$

\begin{tabular}{|c|c|c|c|c|c|c|}
\hline $\begin{array}{l}\text { Lag } \\
\text { (months) }\end{array}$ & $b_{1}$ & $S E M\left(b_{1}\right)$ & $b_{2}$ & $S E M\left(b_{2}\right)$ & $b_{3}$ & $S E M\left(b_{3}\right)$ \\
\hline 9 & 3.6 & 5.5 & $2 \cdot 2$ & 3.4 & 0.8 & 1.7 \\
\hline 10 & -3.6 & $5 \cdot 5$ & $-2 \cdot 2$ & $3 \cdot 5$ & $-1 \cdot 5$ & $1 \cdot 7$ \\
\hline 11 & -0.2 & 5.5 & 1.5 & 3.4 & 1.5 & 1.7 \\
\hline 12 & $-4 \cdot 5$ & 5.5 & -1.8 & 3.5 & -0.3 & 1.7 \\
\hline 13 & $-8 \cdot 3$ & 5.5 & $-5 \cdot 6$ & 3.6 & $-2 \cdot 1$ & 1.8 \\
\hline 14 & -0.0 & $5 \cdot 6$ & -0.2 & 3.5 & $0 \cdot 1$ & 1.7 \\
\hline 15 & $-7 \cdot 2$ & $5 \cdot 6$ & $-4 \cdot 7$ & 3.6 & -1.7 & 1.7 \\
\hline 16 & $10 \cdot 0^{*}$ & $5 \cdot 6$ & $2 \cdot 7$ & $3 \cdot 4$ & -0.8 & 1.7 \\
\hline 17 & $7 \cdot 0$ & $5 \cdot 7$ & 4.8 & 3.5 & $2 \cdot 8$ & 1.7 \\
\hline 18 & $8 \cdot 1 t$ & $5 \cdot 7$ & $7 \cdot 3 \ddagger$ & $3 \cdot 4$ & $4.5 \S$ & 1.7 \\
\hline \multicolumn{7}{|l|}{ Extra-Poisson/ } \\
\hline Poisson ratio & $3 \cdot 6$ & & $2 \cdot 7$ & & $1 \cdot 1$ & \\
\hline
\end{tabular}

$b_{1}=$ logistic regression coefficient of monozygotic twins as a proportion of total twins (allowing for extra-binomial variation); $b_{2}=$ log-linear regression coefficient of monozygotic twins as a proportion of total confinements (allowing for extra-Poisson variation); $b_{3}=l_{0}$-linear regression coefficient of like sex twins as a proportion of total confinements.

${ }^{*} \mathrm{p}=0.08 ; \quad \dagger \mathrm{p}=0.14 ; \quad \neq \mathrm{p}=0.034 ; \quad \S \mathrm{p}=0.01$

\section{Discussion}

We have shown an association between oral contraceptive discontinuation rates and monozygotic twinning 18 months later, which is compatible with the results of other studies. Any assessment of the validity of the findings must take into account several features of our data. We estimated monthly variation in the numbers of women taking the pill prior to conception using the discontinuation rate. However, OC use ceased for a variety of reasons other than to conceive, ${ }^{6}$ which will have diluted any observable association, and we had no information about types of $O C$ used. Twin live- and stillbirths will have been accurately counted in a total population, since birth registration is nearly complete. However, the real dependent variable of interest is twin conceptions, and we were unable to include twin abortions, therapeutic or otherwise. We also do not know the extent to which the phenomenon of the "vanishing twin" may have varied by month or sex. ${ }^{14}$ Moreover, while the sex of twin registrations is accurately known we have only been able to estimate zygosity using Weinberg's method. Its assumptions of $1: 1$ sex ratio, independent determination of the sex of co-twins and similar selective factors including perinatal loss and birth ascertainment among like and unlike sexed dizygotic twins have been questioned, ${ }^{15}$ but these effects are probably small in the Scottish population, and important bias is unlikely. However the monthly estimates of zygosity, based on relatively small numbers of twins, will be somewhat inaccurate and weaken any observable association. We also estimated OC discontinuation rates and twinning by calendar rather than equal lunar months, and although the contraceptive discontinuation rates were seasonally adjusted this will have reduced the precision of the estimated correlation, as will the restriction to monthly intervals rather than weeks, since mean gestational length for twins in 37-38 weeks. ${ }^{16}$

With regard to our analysis, the actual level of significance must take into account the number of implicit significance tests. Multiple significance testing and correlation between the coefficients have opposing effects on the significance level of the correlations. We did not have a strong prior hypothesis about the nature of the relationship except that any lag which existed must be longer than 8 months and probably less than 21 . Thus these findings should be viewed as generating hypotheses, rather than testing prior ones.

Previous studies have provided conflicting results, suggesting variously higher dizygotic twin rates following OC discontinuation, ${ }^{1 P}$ lower overall twinning rates amongst former users compared to non-users but with increased unlike sexed twinning in subgroups, ${ }^{18} 19$ decreased dizygotic twinning rates amongst recent users, ${ }^{20}$ increased monozygous twinning following discontinuation, ${ }^{21}$ and no effect at all. ${ }^{22} 23$ Conclusions in these studies about the importance of the period between discontinuation and conception have also varied. Mechanisms suggested are pituitary inhibition or stimulation for the dizygotic twins or delay in implantation for the monozygotic effect. ${ }^{172425}$ Zygosity was only once determined precisely in these studies, ${ }^{23}$ usually being estimated by Weinberg's rule. Since this must produce complementary errors in estimated mono- and dizygotic twinning, and study design, size and type of $O C$ used have varied considerably since the first investigation, it is perhaps not surprising the results conflict.

In our study, total confinements were significantly related to $O C$ discontinuation rates 13 and 16 months 
previously. Assuming a gestation of 38 weeks, both lags are consistent with findings in previous studies: a 3-4 month median time to conception following pill discontinuation has been described, ${ }^{172627}$ as has a 9 month mean interval to conception following OC discontinuation for singletons, and like sex and unlike sex twins. ${ }^{20}$ The correlation we found with monozygotic twinning at 18 months also corresponds to this latter finding, providing no support for the suggestion of the higher fertility of twin prone mothers, which may be restricted to dizygotic twins. ${ }^{28}$ It has recently been suggested that ovulatory stimulants may be the first identified biological mechanism influencing monozygotic twinning rate, ${ }^{29}$ in addition to their effect on the dizygotic rate. The precise role of artificial induction of ovulation in the trends in twinning rates in Great Britain is as yet unestablished, ${ }^{22}$ but if their use in the infertile population was (becoming) widespread, ${ }^{30}$ the frequency of ensuing multiple births would have an important influence on the trends. However, while we cannot provide a specific biological explanation for the 9 month lag we found between cessation of pill use and increased monozygotic twinning, we find it difficult to see why trends in the use of ovulatory stimulants and subsequent twinning should explain this correlation. We found no correlation at all for the dizygotic rate and no evidence for an increasing secular trend in the monozygotic rate as judged by the absence of (spurious) "seasonal" effects. Accordingly we conclude that the notion that the discontinuation of OCs (or certain types of OC) affects twinning remains plausible but unproven. Further studies are required since potentially large numbers of women are at risk of this occurrence.

We would like to thank David Firth of the Department of Mathematics, Imperial College, London who suggested the approach based on the probability of twins given fertilisation of a single egg. Christine Eckersley of the Department of Community Medicine and General Practice, Oxford, typed the manuscript, and the Scottish twin registration data were provided by Christine Rae of the General Register Office (Scotland).

Address for correspondence and reprints: $\operatorname{Dr}$ M F G Murphy, Department of Community Medicine and General Practice, Radcliffe Infirmary, Oxford OX2 $6 \mathrm{HE}$.

\section{Appendix}

In month $t$ let $\mathrm{m}_{\mathrm{t}}=$ total number of confinements. $r_{t}=$ total number of like sex twins (ie, twice the number of like sex twin pairs)

$=2\left(a_{t}-b_{t}\right)$, where $a_{t}$ and $b_{t}$ are the total number of twin pairs and number of unlike sexed twin pairs respectively in month $t$.

$Y_{t}=$ probability of twins given the fertilisation of a single egg.

$\mathrm{X}=$ probability of a dizygotic twin pregnancy (assumed independent of $t$ ).

Then the expected number of like sex twins in month $t$ is computed as follows: Pregnancies of higher order than twins are disregarded, so if one egg is fertilised with probability $(1-X)$, the probability of twins is $(1-X) Y_{t}$ and the expected number of monozygotic twins is $2 m_{t}(1-X) Y_{t}$. Two fertilised eggs are produced with probability $X$, but only half of these are like sex. Thus the total expected number of like sex twins is

$$
E\left(r_{t}\right)=X m_{t}+2 m_{t}(1-X) Y_{t} \text {. }
$$

We wish to investigate the dependence of $Y_{t}$ on the $O C$ discontinuation rates. Thus we can model the number of like sex twins as a Poisson distribution, with $E\left(r_{t}\right)$ given by equation (1).

We can estimate $X$ by $\Sigma 2 b_{t} / \Sigma m_{t}=0.0052$. Since $X$ i so small, equation (1) can be well approximated by $E\left(r_{t}\right)=2 m_{t} Y_{t}$. Thus we can investigate the dependence of $Y_{t}$ on factors such as the OC discontinuation rate using simple Poisson regression.

In GLIM, to examine the dependence of the probability of monozygotic twinning on the $O C^{-}$ discontinuation rates we regress the monthly count of like sex twins, $r_{t}$, on the $O C$ discontinuation rate using a Poisson model with OFFSET $\log 2 \mathrm{~m}_{\mathrm{t}}$.

\section{References}

${ }^{1}$ James W. Secular changes in dizygotic twinning rates. $J$ Biosoc Sci 1972; 4: 427-34.

2 James W. Second survey of secular trends in twinning rates. J Biosoc Sci 1982; 14: 481-97.

${ }^{3}$ Weinberg W. Beitrage zur Physiologie und Pathologie der Mehrlingsgeburten beim Menschen. Pflugers Arch 1901; 88: $346-430$.

4 James W. Secular changes in twinning rates in England and Wales. Ann Hum Biol 1980; 7: 485-7.

${ }^{5}$ Elwood $\mathrm{J}$. The end of the drop in twinning rates? Lancet 1983; i: 470.

6 James W. Twinning rates. Lancet 1983; i: 934-5.

${ }^{7}$ Bone M. Family planning in Scotland in 1982. A survey carried out on behalf of the Scottish Home and Health Department. London: HMSO, 1985.

${ }^{8}$ Westoff CF, Ryder NB. The contraceptive revolution. Princeton, New Jersey: Princeton University Press, 1977.

${ }^{9}$ Edwards J. The recognition and estimation of cyclic trends. Ann Hum Genet 1961; 25: 83-7.

${ }^{10}$ Baker R. Nelder J. The GLIM system. Release 3. Oxford: Numerical algorithms Group, 1978. 
11 Williams D A. Extra-Binomial variation in logistic linear models. Appl Stat 1982; 31: 144-8.

12 Breslow N. Extra-Poisson variation in loglinear models. Appl Stat 1984; 33: 38-44.

${ }^{13}$ Chatfield C. The analysis of time-series. London: Chapman and Hall, 1975.

${ }^{14}$ Landy H, Keith L, Keith D. The vanishing twin. Acta Genet Med Gemellol 1982; 31: 179-94.

15 Allen G. Errors of Weinberg's difference method. In: Twin research 3: Twin biology and multiple pregnancy. New York: Alan R. Liss, 1981: 71-4.

${ }^{16}$ Saunders M, Dick J, Brown I, McPherson K, Chalmers I, The effect of hospital admission for bed rest on the duration of twin pregnancy: a randomised trial. Lancet 1985; ii: 793-5.

${ }^{17}$ Bracken M. Oral contraception and twinning: an epidemiologic study. Am J Obstet Gynecol 1979; 133: 432-4.

${ }^{18}$ Vessey M, Meisler L, Flavel R, Yeates D. Outcome of pregnancy in women using different methods of contraception. Br J Obstet Gynaecol 1979; 86: 548-558.

19 Rothman K. Fetal loss, twinning and birthweight after oral contraceptive use. $N$ Engl J Med 1977; 297: 468-71.

${ }^{20}$ Hemon D, Berger C, Lazar P. Twinning following oral contraceptive discontinuation. Int J Epidemiol 1981; 10: 319-28.

${ }^{21}$ Macourt D, Stewart P, Zaki M. Multiple pregnancy and fetal abnormalities in association with oral contraceptive usage Aust N Z J Obstet Gynaecol 1982; 22: 25-8.
${ }^{22}$ Webster F, Ellwood J. A study of the influence of ovulation stimulants and oral contraception on twin births in England. Acta Genet Med Gemellol 1985; 34: $105-8$.

${ }^{23}$ Campbell D, Thompson B, Pritchard C, Samphier M. Does the use of oral contraception depress dizygous twinning rates. Acta Genet Med Gemellol (In press).

${ }^{24}$ MacGillivray I. The probable explanation for the falling twinning rate in Scotland. In: Twin research 3: Twin biology and multiple pregnancy. New York: Alan R Liss, 1981: 15-9.

${ }^{25}$ Emery A. Identical twinning and oral contraception. Biology and Society 1986; 3: 23-7.

26 Vessey M, Wright N, McPherson K, Wiggins P. Fertility after stopping different methods of contraception. $\mathrm{Br}$ Med J 1978; i: 265-7.

${ }^{27}$ Bone M. The Family Planning Services: changes and effects. A study carried out on behalf of the Department of Health and Social Security. London: HMSO. 1978.

28 James $\mathrm{W}$. The declines in dizygotic twinning rates and in birthrates. Ann Hum Biol 1975; 2: 81-4.

${ }^{29}$ Derom C, Derom R, Vlietinck R, Van Den Berghe $H$, Thiery $M$. Increased monozygotic twinning rate after ovulation induction. Lancet 1987; i: 1236-8.

${ }^{30}$ Murphy M, Botting B. Twinning rates and social class in Great Britain. Arch Dis Child 1989; 64: 272-4.

Accepted for publication January 1989 